Nouveaux cahiers de la recherche en éducation

\title{
Influence parentale sur le rendement scolaire : comparaison entre des élèves nés au Canada et des élèves nés à l'extérieur du pays
}

\author{
Jean-Jacques Doucet, Anouk Utzschneider et Jimmy Bourque
}

Volume 12, numéro 2, 2009

URI : https://id.erudit.org/iderudit/1017469ar

DOI : https://doi.org/10.7202/1017469ar

Aller au sommaire du numéro

Éditeur(s)

Faculté d'éducation, Université de Sherbrooke

ISSN

1911-8805 (numérique)

Découvrir la revue

Citer cet article

Doucet, J.-J., Utzschneider, A. \& Bourque, J. (2009). Influence parentale sur le rendement scolaire : comparaison entre des élèves nés au Canada et des élèves nés à l'extérieur du pays. Nouveaux cahiers de la recherche en éducation, 12(2), 227-242. https://doi.org/10.7202/1017469ar

\section{Résumé de l'article}

Des études suggèrent un lien entre la participation parentale aux devoirs, le rendement scolaire des élèves et l'appartenance ethnique. Notre hypothèse était que l'influence parentale sur des enfants qui ne sont pas nés au Canada serait plus importante, en sciences, que chez les élèves nés au pays. Nos données provenaient du Programme d'indicateurs du rendement scolaire et contenaient les réponses à des questionnaires soumis à 24502 élèves. Nos analyses nous indiquent des résultats statistiquement significatifs suggérant l'existence de liens entre la réussite en sciences et l'implication des parents dans les devoirs, et entre la réussite des élèves et le jugement de l'importance de la réussite en sciences par les parents. Ces résultats seraient cependant semblables peu importe l'appartenance ethnique.
Tous droits réservés @ Faculté d'éducation, Université de Sherbrooke,

Ce document est protégé par la loi sur le droit d'auteur. L'utilisation des services d'Érudit (y compris la reproduction) est assujettie à sa politique d'utilisation que vous pouvez consulter en ligne.

https://apropos.erudit.org/fr/usagers/politique-dutilisation/ 


\title{
Influence parentale sur le rendement scolaire: comparaison entre des élèves nés au Canada et des élèves nés à l'extérieur du pays
}

\author{
Jean-Jacques Doucet, Anouk Utzschneider et Jimmy Bourque
}

Université de Moncton

Résumé - Des études suggèrent un lien entre la participation parentale aux devoirs, le rendement scolaire des élèves et l'appartenance ethnique. Notre hypothèse était que l'influence parentale sur des enfants qui ne sont pas nés au Canada serait plus importante, en sciences, que chez les élèves nés au pays. Nos données provenaient du Programme d'indicateurs du rendement scolaire et contenaient les réponses à des questionnaires soumis à 24502 élèves. Nos analyses nous indiquent des résultats statistiquement significatifs suggérant l'existence de liens entre la réussite en sciences et l'implication des parents dans les devoirs, et entre la réussite des élèves et le jugement de l'importance de la réussite en sciences par les parents. Ces résultats seraient cependant semblables peu importe l'appartenance ethnique.

Abstract - Studies have suggested that there are links between parental involvement in homework, school performance, and ethnic affiliation. Our hypothesis was that in science, parental influence on pupils born outside Canada is greater than on pupils born in Canada. We obtained our data from the School Achievement Indicators Program. These data contained answers to questionnaires administered to 24,502 pupils. Our analyses point to statistically significant results that suggest links between: pupils' success in science and parental involvement in homework; and pupils' success and parental views of the importance of success in science. However, these results appear to be the same regardless of ethnic affiliation. 


\section{Introduction}

Dans les années 1950, plusieurs études suggéraient que les performances des écoliers étaient davantage reliées à l'appartenance sociale et à l'attitude des parents qu'à l'école (Coleman, Campbell, Hobson, McPartland, Mood, Weinfeld et York, 1966; Institut national d'études démographiques, 1970). Par la suite, les chercheurs ont délaissé le côté parental pour surtout s'intéresser à l'observation du système scolaire en mettant l'accent sur le rôle que jouent l'organisation et le fonctionnement des écoles ainsi que les pratiques d'enseignement. Depuis le milieu des années 1980, l'influence parentale comme moyen pour contrer l'échec scolaire a refait surface au Royaume-Uni et aux États-Unis avec un discours portant sur les relations familles-écoles. Dans ce courant d'idées, le processus d'apprentissage d'un enfant n'est pas uniquement lié aux mécanismes cognitifs mais est aussi fonction d'une dynamique émotive, soit les attitudes et l'intérêt que manifestent les parents à l'égard de l'apprentissage et des résultats scolaires (Sheldon et Epstein, 2005; Van Voorhis, 2001).

Le sujet de l'impact de l'influence parentale sur le rendement scolaire des élèves a été abondamment étudié par les chercheurs. Parmi ceux-ci, on remarque Epstein, dont les travaux ont servi de base à plusieurs études empiriques, dont celles de Van Voorhis (2001) et de Deslandes et Lafortune (2001). Epstein (1995) avance que le soutien aux parents dans leur rôle d'éducateurs est souvent évoqué par les chercheurs comme mesure pédagogique susceptible de contrer les difficultés et, de la sorte, de favoriser la réussite scolaire. De plus, certaines études (Comeau et Claes, 1996; Montandon, 1996) démontrent une relation positive entre la participation parentale et la réussite scolaire des élèves.

Par ailleurs, le sentiment de compétence de l'élève prédit non seulement son rendement, mais également la quantité d'efforts fournis et ses aspirations scolaires (Deslandes et Lafortune, 2001). Or, l'étude de Wigfield, Harold, Freedman-Doan, Eccles, Suk Yoon, Arbreton et Blumenfeld (1997) révèle que le sentiment de compétence de l'élève se bâtit à partir de son rendement antérieur et des rétroactions provenant des agents de socialisation, dont les enseignants et les parents. Enfin, les attentes et les croyances des parents sont de meilleurs prédicteurs du sentiment de compétence de l'élève que ne le sont les notes accordées par les enseignantes et les enseignants (Ibid.).

\section{2. Éléments théoriques}

\subsection{L'apprentissage des sciences}

Dans ce contexte de préoccupations envers la réussite scolaire, depuis une vingtaine d'années, le Canada s'intéresse tout particulièrement à l'importance de l'enseignement des sciences pour former des Canadiennes et des Canadiens capables de s'adapter aux nombreux changements politiques et sociaux d'une société dont les relations entre les personnes sont de plus en plus définies par la technologie. Un rapport du Conseil des sciences du Canada datant de 1984 et intitulé $A$ l'école des sciences: la jeunesse canadienne face à son avenir ainsi que la publication en 1997 du Cadre commun de résultats d'apprentissage en sciences de la nature (Conseil des ministres de l'Éducation du Canada) ne sont pas étrangers à cet intérêt grandissant. On ne peut non plus passer sous silence la publication, en 1996, de La culture scientifique par le Conference Board du 
Canada qui expose l'importance d'enseigner à tous les élèves les sciences de façon stimulante, pertinente et accessible.

D'aucuns sont d'avis que l'apprentissage des sciences est davantage qu'un ensemble de connaissances et de théories complexes et abstraites sur l'univers ou qu'une série de processus orientant la recherche et la découverte scientifiques (Conseil des ministres de l'Éducation, 2005). Sans diminuer l'importance de ces facettes, les auteurs soutiennent que pour faciliter l'apprentissage des sciences chez les élèves, celles-ci doivent faire partie intégrante de leur quotidien. Qui plus est, les élèves apprennent plus facilement s'ils sont confrontés à des expériences pratiques leur permettant d'acquérir des compétences et des connaissances transférables dans leur vie de tous les jours (Ibid.).

Les parents ont nécessairement un rôle à jouer dans l'apprentissage des sciences chez les élèves. Ce sont les familles qui parviennent à renforcer l'importance de l'école, des devoirs à la maison et des activités parascolaires qui permettent l'établissement des capacités scolaires et du sentiment de succès des élèves. Les principaux intéressés, soient les élèves, abondent dans le même sens. À peu près tous les élèves - ordres élémentaire, intermédiaire et secondaire - veulent que leur famille soit un partenaire mieux informé au sujet de l'école et qu'elle soit disposée à prendre un rôle actif dans les communications qui lient la maison et l'école (Epstein, 1995). À ce même sujet, il est aussi mentionné que «selon la perception des adolescents, il existe une relation positive entre la participation parentale dans des activités d'apprentissage à domicile et le rendement en mathématique» (Deslandes et Lafortune, 2001, p. 649).

Bien que conscients de leur responsabilité à l'égard de leurs enfants - toutes les enquêtes réalisées des deux côtés de l'Atlantique indiquent que, contrairement à ce que pensent un certain nombre d'enseignants, les parents investissent beaucoup dans la vie scolaire des enfants (Comeau et Salomon, 1994; Saint-Laurent, Royer, Hébert et Tardif, 1994) - certains parents ne se sentent pas toujours à la hauteur par rapport à l'influence qu'ils exercent au niveau du rendement scolaire. Cela est particulièrement vrai dans l'apprentissage des sciences. Deslandes et Lafortune (2001) rapportent que cette situation se présente aussi en mathématiques. Parmi les raisons évoquées expliquant le malaise des parents, on remarque que les démarches de réflexion sont maintenant différentes de celles retrouvées dans les façons d'apprendre traditionnelles: les approches sont différentes de celles que beaucoup de parents ont connues (Dodd et Konzal, 2000).

Il ne faut cependant pas généraliser le rapport des parents face au rendement scolaire des enfants. À cet égard, Lareau (1989) mentionne qu'il faut tenir compte des caractéristiques des parents, notamment de leur origine sociale et ethnique, en fonction de leurs ambitions pour la scolarité de leur enfant. La dimension de l'origine ethnique offre donc ici une avenue qui mérite d'être investiguée puisque, jusqu'à ce jour, il ne semble pas que la relation entre l'origine ethnique et l'aide parentale dans les devoirs ait été très bien comprise.

\subsection{Une comparaison au plan international}

Dans ce sens, la performance scolaire des élèves asiatiques suscite depuis quelques années l'intérêt des chercheurs, qui ont été à même d'observer des écarts importants dans le cadre de comparaisons internationales ou encore de comparaisons entre des élèves asiatiques et des élèves natifs du pays (Glasgow Winters, 1993). Selon Comeau et Claes (1996), le niveau de succès des 
élèves asiatiques est relié aux aspirations élevées des parents asiatiques ainsi qu'aux croyances qu'ils leur transmettent, à savoir que l'effort soutenu se traduit nécessairement par des résultats élevés.

Les études internationales nous révèlent que les élèves états-uniens sont loin derrière leurs pairs internationaux en mathématiques et en sciences (Pong, 1997). Il est aussi admis que les écarts au niveau de la nation états-unienne ne sont pas uniquement dus à des différences dans les systèmes éducatifs, mais que ces écarts sont le reflet de différences culturelles, de valeurs transmises et de comportements (Chen, 2001).

Une étude menée par Chen (Ibid.) tend à démontrer les différences dans les attitudes envers les sciences parmi des parents et des étudiants états-uniens, des Sino-Américains et des parents et des étudiants chinois. L'étude visait aussi à comparer les trois groupes culturels en fonction des attentes des parents envers la performance en science de leurs enfants au secondaire. Les résultats de l'étude ont démontré que les parents ainsi que les étudiants chinois avaient des attitudes plus positives envers l'apprentissage des sciences que leurs confrères états-uniens. Il appert que les parents chinois mettent plus d'accent sur l'amélioration de soi, établissent des critères plus élevés et aident plus souvent leurs enfants dans l'apprentissage des sciences que les parents états-uniens. Les attitudes des Sino-Américains sont apparues comme une démonstration de l'influence de leur héritage chinois et de la culture américaine. D’une façon générale, une corrélation positive élevée a été trouvée entre l'attitude des parents et des étudiants envers l'apprentissage des sciences.

\subsection{Participation parentale au plan international}

Dans certains pays, la participation parentale à l'éducation des enfants est considérée comme un droit. Des pays membres de l'Organisation de coopération et de développement économique (OCDE) ont rédigé ce droit en loi. C'est le cas notamment pour la France, l'Allemagne et le Danemark (Kelley-Laine, 1998). Les droits appellent aux responsabilités et ces responsabilités prennent la forme de trois activités en termes de participation parentale dans les pays de l'OCDE (Ibid.): 1) le soutien psychosocial - le soutien que les écoles fournissent aux familles, peut être sous la forme d'éducation parentale; 2) la communication entre l'école et la maison et 3) la participation parentale au travail de l'école, dans la classe et à la maison.

De nombreuses études ont mis en évidence les liens positifs entre la participation des parents et le rendement scolaire des élèves (Ong, Phinney et Dennis, 2006; Deslandes et Bertrand, 2004 ; Dornbusch et Ritter, 1992; Henderson et Berla, 1994; Henderson et Mapp, 2002; Potvin, Deslandes, Beaulieu, Marcotte, Fortin, Royer et Leclerc, 1999).

\subsection{Pratiques de participation parentale}

Dans le contexte scolaire, lorsqu' on parle de participation parentale, on entend surtout la façon dont un parent prend part à la scolarisation de ses enfants (Van Peteghem, 2006). Le rendement scolaire se définit comme le « degré de réussite d'un sujet ou d'un ensemble de sujets en regard des objectifs spécifiques des divers programmes d'études ou encore comme la qualité et quantité du travail d'un sujet, ou un groupe de sujets, en situation pédagogique» (Legendre, 2005, p. 1177). 
Sheldon et Epstein (2005) parlent d'implication parentale en faisant référence à un vaste nombre d'activités et de connexions entre l'école, les familles et la communauté. Selon Epstein (1995), il existe six types d'implication dans un programme de partenariat entre l'école, la famille et la communauté. Parmi ceux-ci, on retrouve la mise en place d'un soutien familial, mais aussi une amélioration de la communication bilatérale entre l'école et les parents, la promotion du bénévolat chez les parents à l'école et à la maison, de l'aide parentale dans les travaux, de l'implication des parents dans la prise de décision à l'école, et de la collaboration avec la communauté.

Selon Potvin et al., (1999), les formes de participation parentale peuvent varier. Elles comprennent, par exemple, la préparation de l'enfant à l'école et l'accueil à son retour, la supervision, le suivi et l'engagement actif dans les travaux scolaires, la contribution à la motivation de l'enfant, les interactions avec l'école, le bénévolat à l'école et la participation à des comités de parents, des réunions et des assemblées générales (Deslandes et Bertrand, 2004).

Selon Montandon (1996), certains auteurs tiennent compte d'autres indicateurs pour définir l'implication parentale: les discussions au sujet des enseignants, des camarades ou de la vie de classe, la suite que les parents donnent aux activités scolaires, l'organisation du temps et de l'espace à la maison dans le but de favoriser les apprentissages ou encore, sur le plan plus subjectif, les aspirations des parents concernant l'avenir de leurs enfants.

\subsection{Devoirs à la maison}

Parmi les activités de participation parentale, les devoirs, en tant qu'activité quotidienne impliquant des interactions particulières entre le parent et l'enfant, occupent une place importante dans la vie familiale (Giasson et Saint-Laurent, 2004). On décrit les devoirs comme des «travaux que l'élève doit exécuter en dehors de l'horaire régulier de l'école, habituellement à la maison, dans le but d'approfondir et de consolider des apprentissages récents » (Legendre, 2005, p. 393). Selon MacBeth (1989), le travail scolaire à domicile (les devoirs) peut constituer une dimension importante de l'implication parentale. D'après Xu (2004), les devoirs à la maison sont souvent considérés comme un véhicule important pour développer de bonnes habitudes de travail. Humbeeck, Lahaye, Balsamo et Pourtois (2006) avancent que les modalités de prise en charge des devoirs de l'enfant par les parents conditionnent le rapport à l'école. D'après ces chercheurs, les devoirs à la maison facilitent, pour les enfants, l'acquisition de compétences puisqu'ils permettent aux parents de repérer l'endroit où les apprentissages n'ont pas été réalisés. Une fois le problème identifié, des stratégies de régulation peuvent être mises de l'avant. Dans cette perspective, les devoirs sont considérés comme un instrument de communication entre l'école et la famille ainsi qu'un outil pédagogique.

\subsection{Soutien affectif}

Sur le plan affectif, les manifestations d'encouragement des parents qui soutiennent, complimentent et offrent leur aide sont associées à de meilleurs résultats scolaires ou à une amélioration de la performance scolaire (Dornbusch et Ritter, 1992). Deslandes (1996) a identifié cinq dimensions reliées à la participation parentale influençant le rendement scolaire. On remarque parmi ces dimensions le soutien affectif rendu possible par la communication entre parents et enseignants, les interactions axées sur le quotidien scolaire, la communication entre les parents et l'école, de même que la communication entre parents et adolescents. 
D'après Kelley-Laine (1998), les parents sont les principaux responsables des premiers stades de socialisation des enfants. Ils sont non seulement responsables de l'établissement chez leurs enfants des bases cognitives, mais aussi des bases émotives sur lesquelles l'école et la communauté peuvent construire.

\subsection{Dimension culturelle et ethnique de la participation parentale}

L'éducation est un sujet important lorsque l'on s'intéresse à la diversité ethnique à l'intérieur d'une société car elle constitue un facteur clé de la reproduction ou de la réduction des inégalités ethniques (Coll, Lamberty, Jenkins, McAdoo, Crnic, Wasik et Vazquez Garcia,1996). Tant la famille que l'école sont intimement impliquées dans l'éducation des enfants. Cependant, la façon avec laquelle elles se conduisent et partagent leurs responsabilités varie d'un pays à l'autre (Kelley-Laine, 1998). Des recherches ont démontré que l'implication parentale varie grandement en fonction de l'appartenance ethnique et, ainsi, tendent à expliquer les différents niveaux de réussite des élèves (Morrison Gutman et Midgley, 2000).

Il est donc difficile d'effectuer des généralisations au sujet de la relation entre l'implication parentale et la réussite scolaire des élèves, puisqu'il s'agit d'une relation complexe. Il ressort en effet de la lecture de nombreux articles sur le sujet que les résultats concernant la réussite des élèves varient selon le type d'implication parentale, la perspective de mesure de la réussite (point de vue des élèves ou des parents), les mesures de réussite utilisées, le sujet scolaire, le statut socioéconomique des parents et, finalement, l'origine ethnique.

Les parents d'origines aussi diverses que l'Amérique centrale, l'Asie du Sud-Est continentale, les Caraïbes, l'Europe et l'Inde accordent une très grande importance au succès académique de leurs enfants (Fuligni, 1997). Ces parents croient que l'éducation est la meilleure façon pour leurs enfants d'améliorer leur statut. Les encouragements et les aspirations des parents immigrants sont probablement les éléments les plus importants de l'influence parentale sur l'éducation de leurs enfants (Ibid.).

Selon Okagaki et Frensch (1998), les parents d'origine asiatique ont de plus hautes attentes envers la réussite scolaire de leurs enfants. Toutefois, ces auteurs ne notent pas de différence au plan de la fréquence de l'aide aux devoirs à l'intérieur des différents groupes ethniques. Selon Chen et Stevenson (1995), les élèves asiatiques seraient plus susceptibles que les élèves étatsuniens d'avoir des parents avec des attentes élevées envers leur rendement scolaire ainsi que des convictions à l'effet que le succès académique n'arrive pas sans un dur labeur. Des recherches comparant des parents japonais et états-uniens (Holloway, 1988) ont indiqué que les parents japonais mettent davantage d'emphase sur le rôle de l'effort dans la réussite scolaire que ne le font les parents états-uniens. Ainsi, leurs attentes envers les résultats scolaires de leurs enfants ne sont pas affectées par la perception qu'ils ont des habiletés de leurs enfants.

Eccles et Harold (1996) ont trouvé que les parents afro-américains démontraient une plus grande implication dans les activités scolaires à la maison comparativement aux parents «blancs» qui, de leur côté, étaient davantage portés à faire du bénévolat ou à participer aux activités de l'école. Hong et Ho (2005) ont, quant à eux, trouvé que les aspirations parentales au sujet de l'école affectent davantage la réussite scolaire des adolescents blancs que celle des adolescents afro-américains ou latino-américains. 
À la suite de leurs recherches auprès de familles de réfugiés de l'Asie du Sud-Est continentale, Caplan, Choy et Withmore (1992) ont conclu que l'aide aux devoirs par les parents n'était pas aussi importante que le climat général établi par les parents et dans lequel l'éducation, l'étude et les bonnes notes sont d'une grande importance.

Même si la réussite scolaire est souvent plus faible chez les élèves latino-américains que chez les élèves d'origine européenne américaine aux États-Unis, une étude de Stevenson, Chen et Uttal (1990) révèle que dans un échantillon de 1000 mères ayant des enfants fréquentant l'école élémentaire, les mères d'origine latino-américaine accordaient plus d'importance à la réussite académique que les mères d'origine européenne américaine. Dans cet ordre d'idées, Martinez, DeGarmo et Eddy (2004), de même que Schneider et Ward (2003) avancent que le support parental et l'interdépendance familiale ont été identifiés comme des éléments clé de la réussite scolaire parmi les latino-américains. De plus, d'après Ong, Phinney et Dennis (2006), les étudiants latino-américains avec plus de ressources psychologiques et familiales réussissent mieux au plan scolaire.

Ceballo (2004) soulève le fait que les parents latino-américains issus de la classe ouvrière voient l'éducation comme le moteur le plus important de la mobilité sociale et économique. Toutefois, ils démontrent leur soutien à l'éducation d'une façon différente de celle des parents latino-américains plus éduqués. En effet, ces derniers ont davantage tendance à aider leurs enfants dans leurs devoirs et à interagir avec l'école. Chez les immigrants plus pauvres, le très grand soutien donné par les parents pour l'éducation de leurs enfants reflète la croyance que l'éducation est le «billet qui permettra l'atteinte du rêve américain», celui qui permet de passer de la classe inférieure à la classe supérieure grâce au travail acharné et à la détermination (Fuligni, 1997; Stevenson, Chen et Uttal, 1990). Les parents offrent ainsi leur soutien à leurs enfants même s'ils se sentent mal équipés pour les aider, particulièrement en lecture et en mathématiques, en raison notamment du fait qu'ils ne sont pas à l'aise avec la langue anglaise, de leur connaissance limitée du système scolaire étatsunien et en raison d'horaires de travail souvent très exigeants (Okagaki et Frensch, 1998).

Il semble aussi que chez les étudiants latino-américains du collégial, l'interdépendance familiale soit associée à un fort désir de réussir au niveau scolaire dans le but de dédommager les parents pour les sacrifices qu'ils ont fait en immigrant aux États-Unis (Phinney, Dennis et Osorio, 2006). Enfin, Arrellano et Padilla (1996) lient le soutien parental à l'intensité des liens familiaux.

\section{Objectif et hypothèses}

Les études antérieures nous amènent à penser que la diversité ethnoculturelle aurait une influence sur les pratiques parentales de soutien aux activités scolaires de leurs enfants, qui, à son tour, affecterait le rendement et la réussite scolaires des élèves. En poursuivant notre réflexion, il est à se demander s'il existe une «culture d'apprentissage» des parents et des élèves canadiens qui se démarque des familles des autres cultures de par le monde. Cette étude vise donc à examiner si les élèves qui ne sont pas nés au Canada sont influencés par leurs parents dans leur rendement et réussite scolaires en sciences d'une manière différente que ne le sont les enfants nés au Canada.

Une hypothèse générale peut ici être formulée à la suite de la recension présentée plus haut: la fréquence de l'implication des parents dans la réalisation des devoirs de sciences de leurs enfants aura un effet différent sur le rendement scolaire en sciences des enfants qui ne sont pas nés au Canada et des natifs du pays. De là, deux hypothèses spécifiques peuvent être proposées en cernant 
plus précisément les pratiques parentales: 1) le temps consacré aux devoirs a un effet différent sur le rendement scolaire en sciences des enfants qui ne sont pas nés au Canada et des natifs du pays et 2) les jugements et les encouragements des parents auront une influence différente sur le rendement scolaire des élèves qui ne sont pas nés au Canada et des natifs du pays.

\section{Méthode}

\subsection{Type de recherche et collecte de données}

La présente recherche est de type empirique exploratoire (Van der Maren, 1996) et adopte un devis non expérimental (Johnson et Christensen, 2004). La méthode de collecte de données est le questionnaire d'enquête. Le questionnaire utilisé est issu du Programme d'indicateurs du rendement scolaire (PIRS) - Évaluation en sciences III (Conseil des ministres de l'éducation du Canada, 2004) qui, lui-même, fait partie d'un programme cyclique d'évaluations pancanadiennes portant sur le rendement des élèves en lecture, en écriture, en mathématiques et, bien entendu, en sciences. Les instruments utilisés dans toutes les évaluations du PIRS ont été conçus par les éducatrices et éducateurs francophones et anglophones qui travaillaient ensemble dans le but de prévenir les biais attribuables à la langue. Une analyse linguistique de chaque question et de chaque problème en français et en anglais fut aussi réalisée afin de s'assurer que les items fonctionnaient de la même manière en français et en anglais (Ibid.). L'instrument porte sur les résultats en sciences, le milieu familial des élèves (dont le soutien parental), leur perception de l'école et des sciences, leurs aspirations scolaires et professionnelles, leurs activités extrascolaires et les raisons de leur réussite et de leur échec. Il comporte également des questions sur les méthodes et les ressources pédagogiques utilisées.

\section{2 Échantillon}

L'échantillon aléatoire est constitué de 24502 élèves, soit 13021 élèves de 13 ans et 11481 élèves de 16 ans. L'échantillon provenait du Canada, soit de l'ensemble des dix provinces et de deux des trois territoires, le Nunavut n'ayant pas participé à l'évaluation.

\subsection{Variables et indicateurs}

En lien avec notre première hypothèse spécifique, on retrouve comme première variable indépendante le lieu de naissance: la réponse à la question «Es-tu né au Canada?», à laquelle l'élève répond par oui ou non. La deuxième variable indépendante est l'implication des parents dans la réalisation des devoirs en sciences : la réponse à la question «À quelle fréquence toi et tes parents ou tuteurs faitesvous ensemble tes devoirs de sciences?» Il s'agit ici d'une variable ordinale à quatre niveaux.

En ce qui concerne la deuxième hypothèse spécifique, outre le lieu de naissance, on retrouve les jugements des parents envers la réussite en sciences: la réponse à la question «Comment les personnes suivantes jugent-elles ta réussite en sciences?... a) Parents », dont la réponse est choisie parmi une échelle d'importance à quatre niveaux.

Le rendement scolaire en sciences, qui représente la variable dépendante, est mesuré par le résultat final au test uniformisé de sciences, administré dans le cadre du programme PIRS. 


\subsection{Méthodes d'analyses}

Dans le but de vérifier nos hypothèses de départ, nous avons réalisé deux analyses de variance factorielles univariées. L'estimateur de la taille de l'effet utilisé est oméga-carré (non biaisé puisqu'il prend en compte la part de variance attribuée à l'erreur).

\section{Résultats}

Préalablement à l'analyse des résultats, nous avons utilisé la procédure WEIGHT afin de pondérer statistiquement les données selon le lieu de naissance. Cette méthode permet de s'assurer que les résultats ne soient pas faussés par la grande différence de taille entre les groupes comparés.

\subsection{Analyses descriptives}

Le résultat moyen au test de sciences, pour l'échantillon total $(\mathrm{N}=24983)$, est de $51,46 \%$, la médiane étant de $51,28 \%$. On observe aussi que le plus bas résultat obtenu est $0 \%$ alors que le plus haut s'élève à $94 \%$. L'écart-type est assez élevé à $12 \%$. De plus, l'asymétrie $(-0,028)$ et la voussure $(-0,257)$ suggèrent que la distribution ne s'écarte pas de façon importante de la courbe normale. La distribution des résultats des élèves qui n'ont pas indiqué leur pays de naissance n'est pas distincte de celle de ceux qui ont donné cette information et s'avère aussi répartie normalement.

Pour les élèves qui sont nés au Canada, l'asymétrie $(0,033)$ et la voussure $(-0,272)$ démontrent une distribution qui semble normale. On remarque une moyenne de $52 \%$, une médiane de $51 \%$ et un écart-type de 0,119. Quant aux élèves qui ne sont pas nés au Canada, la distribution semble aussi normale (asymétrie : 0,004; voussure : $-0,180$ ). On remarque une moyenne de $52 \%$, identique à celle des élèves qui ne sont pas nés au Canada, une médiane de $51 \%$ et un écart-type de 0,125 .

Le tableau 1 révèle que les parents sont généralement absents des devoirs et cela autant pour les enfants qui sont nés au Canada que ceux qui ne le sont pas. Il est cependant important de souligner que les données ne nous informent pas sur le besoin des élèves à obtenir l'appui des parents dans les devoirs en sciences. En effet, il est tout à fait plausible de penser que ces élèves n'ont tout simplement pas besoin de l'aide de leur parent pour compléter de façon efficace leurs devoirs en sciences ou encore que les parents ne peuvent pas les aider efficacement dans leurs devoirs.

Tableau 1

Fréquence de l'aide aux devoirs par les parents

\begin{tabular}{|l|c|c|c|}
\hline \multirow{2}{*}{} & \multicolumn{2}{|c|}{ Né(e) au Canada } & \multirow{2}{*}{ Total } \\
\cline { 2 - 3 } & Oui & Non & $70,8 \%$ \\
\hline Rarement ou jamais & $70,8 \%$ & $70,6 \%$ & $19,7 \%$ \\
\hline Quelques fois par mois & $19,8 \%$ & $18,0 \%$ & $8,1 \%$ \\
\hline Quelques fois par semaine & $8,0 \%$ & $9,5 \%$ & $1,4 \%$ \\
\hline Presque tous les jours & $1,4 \%$ & $1,9 \%$ & \\
\hline
\end{tabular}


En ce qui concerne le jugement des parents envers la réussite en sciences, le tableau 2 indique qu'une forte majorité de parents juge importante la réussite en sciences de leur enfant, et ce, tant pour les élèves qui sont nés au Canada que ceux qui sont nés à l'extérieur du pays.

Tableau 2

Jugement des parents envers la réussite en sciences

\begin{tabular}{|l|c|c|c|}
\hline \multirow{2}{*}{} & \multicolumn{2}{|c|}{ Né(e) au Canada } & \multirow{2}{*}{ Total } \\
\cline { 2 - 3 } & Oui & Non & $2,1 \%$ \\
\hline Pas importante du tout & $2,1 \%$ & $2,7 \%$ & $8,0 \%$ \\
\hline Peu importante & $7,9 \%$ & $8,9 \%$ & $50,4 \%$ \\
\hline Importante & $51,0 \%$ & $41,3 \%$ & $39,4 \%$ \\
\hline Très importante & $39,0 \%$ & $47,1 \%$ & \\
\hline
\end{tabular}

\subsection{Effet de l'implication dans l'aide aux devoirs et du jugement quant à la réussite en sciences}

Une ANOVA factorielle univariée ( 2 X 4 X 4) indique un effet significatif du plan factoriel global $(\mathrm{F}[13]=55,393, \mathrm{p}=0,000)$, qui expliquerait $2,9 \%$ de la variance du résultat au test de sciences. Cet effet s'avère petit selon le critère de Cohen $\left(\omega^{2}=0,029\right)$. Quant aux effets individuels, seul celui du lieu de naissance n'est pas significatif $(\mathrm{F}[1]=2,920, p=0,087)$, avec une taille d'effet pratiquement nulle $\left(\omega^{2}=0,000\right)$. L'interaction entre les deux variables indépendantes (l'implication dans les devoirs et le lieu de naissance) aurait un effet significatif ( $\mathrm{F}[3]=3,598, p=$ $0,013)$ mais négligeable $\left(\omega^{2}=0,000\right)$. La même chose se répète pour l'interaction des deux variables indépendantes «lieu de naissance» et «jugements des parents envers la réussite en sciences» $(\mathrm{F}[3]=9,135, p=0,000)$ avec un effet négligeable $\left(\omega^{2}=0,001\right)$. La figure 1 suggère que, pour les deux groupes, l'augmentation de la fréquence de l'implication des parents dans l'aide aux devoirs est associée à une baisse des résultats au test de sciences.

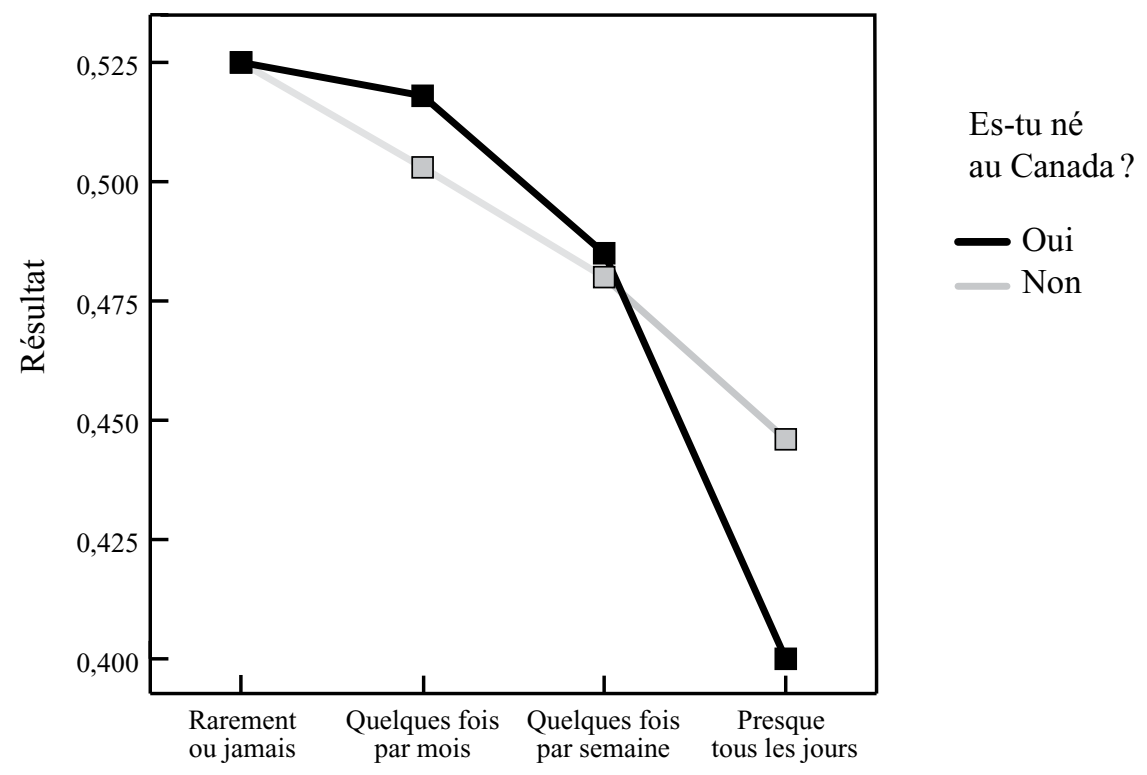

Figure 1 - Relation entre l'aide des parents aux devoirs et les résultats en sciences 
Par ailleurs, la figure 2 révèle que plus la réussite en sciences est jugée importante par les parents, plus les résultats scolaires en sciences sont élevés. Ceci est particulièrement vrai pour les élèves qui ne sont pas nés au Canada.

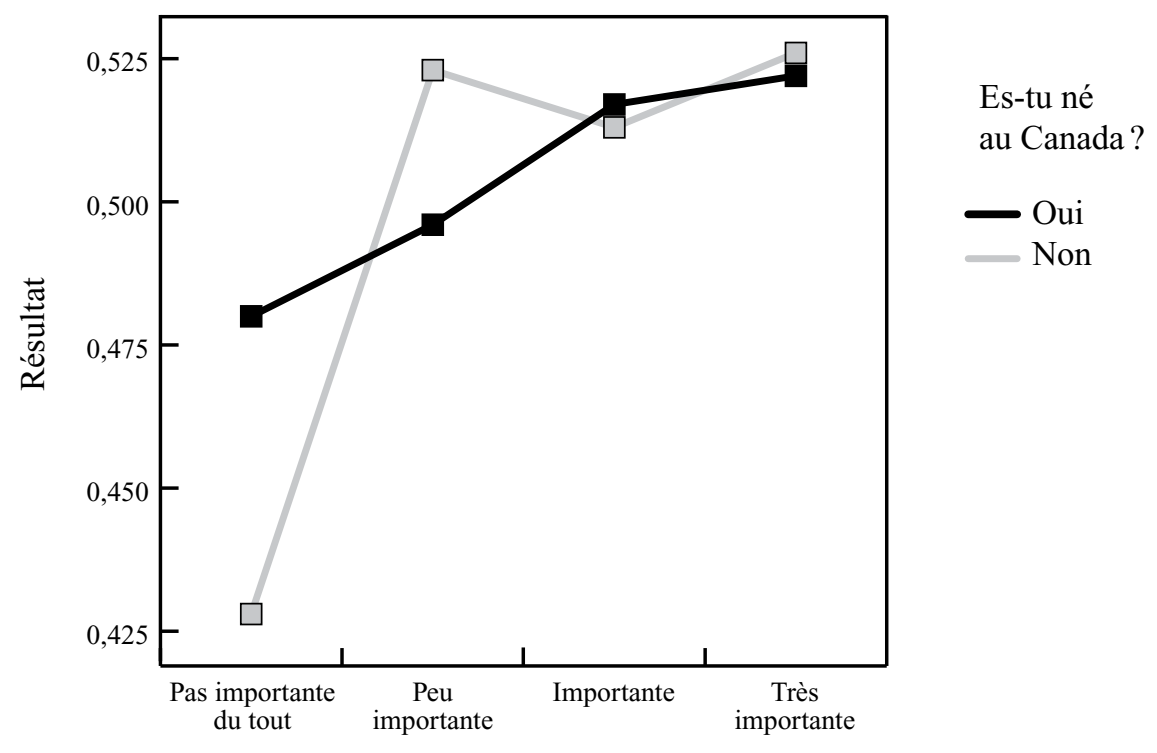

Figure 2 - Jugement des parents en relation avec la réussite en sciences

\section{Discussion}

Cette étude avait pour objectif de déterminer si les élèves qui ne sont pas nés au Canada étaient influencés par leurs parents dans leur rendement et réussite scolaires en sciences d'une manière différente que les enfants nés au Canada. Cet objectif se rattache au besoin d'augmenter le rendement scolaire des élèves canadiens compte tenu des défis à relever en matière de technologie et de l'évolution marquante du marché du travail. Ainsi, la recherche visait à explorer l'effet des pratiques parentales des parents étrangers et canadiens dans le soutien offert à leurs enfants.

En termes de résultats en sciences, les statistiques descriptives démontrent que les moyennes sont les mêmes, que l'on soit né au Canada ou non. L'implication des parents dans la réalisation des devoirs de sciences semble plus importante chez les élèves démontrant de la difficulté que chez les élèves qui obtiennent de meilleurs résultats. On peut supposer que ces résultats indiquent que les élèves éprouvant plus de difficultés réclament davantage d'aide à leurs parents ou encore que les parents sont plus enclins à s'impliquer dans la réalisation des devoirs de leurs enfants lorsque ceux-ci démontrent des difficultés. Par ailleurs, tel que mentionné précédemment, il est tout à fait plausible de penser que les élèves dont les parents s'impliquent peu dans les devoirs n'ont tout simplement pas besoin de l'aide de leur parent pour compléter de façon efficace leurs devoirs en sciences. Au plan méthodologique, l'absence de devis expérimental classique fait que, bien que nous constations un lien entre les variables, il nous est impossible d'établir une relation causale. De toute façon, l'ampleur des effets observés est pratiquement nulle. La signification statistique ne semble donc atteinte qu'en raison de la grande puissance que confère à nos tests un échantillon imposant. 
Par ailleurs, il ne semble pas qu'il existe de différence significative entre l'implication parentale chez les élèves nés au Canada et ceux qui sont nés en dehors du pays, ni sur la réussite en sciences engendrée par cette implication. Ceci rejoint les résultats obtenus par Okagaki et Frensch (1998) qui ne notaient pas non plus de différence au plan de la fréquence de l'aide aux devoirs à l'intérieur des différents groupes ethniques. Il semble même que l'implication des parents dans la réalisation des devoirs ait ici un effet positif légèrement plus prononcé chez les élèves nés au Canada. Cette homogénéité relative des résultats est certainement en grande partie due au fait que les élèves issus de toutes les origines ethniques sont confondus en une seule catégorie. Certaines recherches ont en effet déjà démontré que l'implication parentale varie en fonction de l'appartenance ethnique (Morrison Gutman et Midgley, 2000). Il serait donc intéressant de réaliser une étude sur l'implication des parents dans les devoirs et la réussite en sciences en ayant en main des données plus précises quant à l'origine ethnique des élèves.

D'après les résultats obtenus, il semble également que l'importance accordée à la réussite en sciences par les parents soit associée à l'obtention de meilleurs résultats à l'épreuve de contrôle. Toutefois, les résultats doivent être interprétés avec précaution étant donné le faible degré d'association entre les variables (la taille des effets observés est pratiquement nulle). Comme dans le cas de l'aide aux devoirs, le fait que les élèves issus de différents groupes ethniques soient confondus en une seule et même catégorie fait qu'il est plus difficile de tirer des conclusions précises. Il serait aussi intéressant de documenter la façon dont se traduit de façon concrète l'importance accordée par les parents à la réussite en sciences.

\section{Conclusion}

Ce travail démontre, de par l'abondance des écrits scientifiques, l'importance indéniable du rôle des parents dans l'apprentissage scolaire des enfants. Bien que les écrits démontrent que les parents s'investissent beaucoup dans la vie scolaire de leurs enfants, il est important de nuancer nos résultats en ce sens qu'ils ne portaient que sur une pratique. Il existe effectivement d'autres façons, pour un parent, de s'impliquer dans la vie scolaire de son enfant. Les écrits nous informent également sur le fait que les types d'investissement se font tant sur les plans cognitifs qu'affectifs. La réussite en science à l'école suscite présentement un intérêt pour les parents et la société en général. Étant donné l'influence des parents dans le rendement scolaire, ceux-ci peuvent jouer un rôle dans la réussite en sciences. Des comparaisons au niveau international ont permis d'observer des écarts de performance entre des élèves de divers pays.

À partir de ces constats, la présente étude avait pour objectif de déterminer si les élèves qui ne sont pas nés au Canada sont influencés par leurs parents dans leur rendement et réussite scolaire d'une manière différente que les enfants nés au Canada. D'un point de vue théorique, cette recherche contribue ainsi, modestement, à une meilleure compréhension de l'influence des parents sur la réussite scolaire des enfants en tenant compte de la diversité culturelle qui caractérise le Canada.

Les résultats moyens en sciences des élèves qui sont nés au Canada sont les mêmes que ceux qui sont nés à l'extérieur du pays. Il est aussi suggéré que l'implication des parents dans la réalisation des devoirs en sciences semble plus importante chez les élèves démontrant de la difficulté. Cependant, il est possible que les parents dont les enfants n'ont pas de difficulté en sciences soient désireux de s'impliquer, mais que les élèves n'en ressentent tout simplement pas le besoin. Il est 
aussi important de souligner le fait qu'il soit possible que certains parents n'ont tout simplement pas la compétence pour s'impliquer efficacement dans les devoirs des enfants. Il ne semble pas y avoir de différence significative entre l'implication parentale chez les élèves nés au Canada et ceux qui sont nés à l'extérieur du pays. Il nous est apparu que l'importance accordée à la réussite en sciences par les parents soit souvent associée à l'obtention de meilleurs résultats à l'épreuve de contrôle. Il existe aussi une relation entre le jugement de l'importance de la réussite en sciences et la qualité d'accompagnement donné par les parents mais, comme démontré dans la discussion, les deux ne vont pas nécessairement de pair.

L'appui parental peut cependant s'avérer plus subtil que ne le sont les encouragements ou l'aide dans les devoirs. En effet, le climat familial pourrait influer sur le rendement scolaire de l'enfant. Une difficulté rencontrée dans la présente étude est l'absence de données quant au lieu exact de naissance de l'enfant. En effet, il est plus difficile de tirer des conclusions étant donné l'impossibilité de comparer les élèves en fonction de diverses origines ethniques (pas seulement le fait d'être né ou non au Canada). Cette étude ouvre donc la voie vers d'autres avenues de recherche et s'y dégage l'intérêt de mieux comprendre le rôle des parents dans la réussite scolaire de leurs enfants en contexte multiculturel.

\section{Références}

Arellano, A.R. et Padilla, A.M. (1996). Academic invulnerability among a select group of Latino university students. Hispanic Journal of Behavioral Sciences, 18, 485-507.

Caplan, N., Choy, M.H. et Withmore, J.K. (1992). Indochinese refugee families and academic achievement. Scientific American, 266(2), 36-42.

Ceballo, R. (2004). From Barrios to Yale: The role of parenting strategies in latino families. Hispanic Journal of Behavioral Sciences, 26(2), 171-186.

Chen, H. (2001). Parents' attitudes and expectations regarding science education: Comparaisons among American, Chinese-American, and Chinese families. Journal of Adolescence, 36(142), 305-313.

Chen, C. et Stevenson, H.W. (1995). Motivation and Mathematics Achievement: A Comparative Study of AsianAmerican, Caucasian-American and East Asian High School Students. Child Development, 66(4), $1215-1234$.

Coleman, J.S., Campbell, E.Q., Hobson, C.J., McPartland, J., Mood, A.M., Weinfeld, F.D. et York, R.L. (1966). Equality of educational opportunity. Washington, DC: U.S. Department of Health, Education and Welfare, Office of Education.

Coll, C.G., Lamberty, G., Jenkins, R., McAdoo, H.P., Crnic, K., Wasik, B.H. et Vazquez Garcia, H. (1996). An intergrative model for the study of the developmental competencies in minority children. Child Development, 67, 1891-1914.

Comeau, J. et Claes, M. (1996). L'école et la famille: deux mondes? Lien social et politiques - RIAC, 35, 75-85.

Comeau, J. et Salomon, A. (1994). La participation à l'école: une recherche de sens pour les intervenants. Laval: Agence d'Arc.

Conference Board du Canada (1996). La culture scientifique au travail. Ottawa: Le Conference Board du Canada.

Conseil des ministres de l'éducation du Canada (1997). Cadre commun de résultats d'apprentissage en sciences de la nature. Toronto: Conseil des ministres de l'Éducation.

Conseil des ministres de l'Éducation du Canada (2005). Programme d'indicateurs du rendement scolaire PIRS, Sciences III, 2004. Toronto: Conseil des ministres de l'Éducation du Canada.

Conseil des sciences du Canada (1984). À l'école des sciences: la jeunesse canadienne face à son avenir. Rapport 36. Ottawa: Conseil des sciences du Canada.

Deslandes, R. (1996). Collaboration entre l'école et la famille : influence du style parental et de la participation parentale sur la réussite scolaire au secondaire. Thèse de doctorat en psychopédagogie, Université Laval, Québec. 
Deslandes, R. et Bertrand, R. (2004). Motivation des parents à participer au suivi scolaire de leur enfant au primaire. Revue des sciences de l'éducation, $X X X(2), 411-433$.

Deslandes, R. et Lafortune, L. (2001). La collaboration école-famille dans l'apprentissage des mathématiques selon la perception des adolescents. Revue des sciences de l'éducation, XXVII(3), 649-669.

Dodd, A.W. et Konzal, J.L. (2000). Parents and educators as partners. High School Magazine, 7(5), 8-13.

Dornbusch, S.M. et Ritter, P.L. (1992). Home-school processes in diverse ethnic groups, social classes and family structures. In S.L. Christenson et J.C. Conoley (dir.), Home-school collaboration: Enhancing children's academic and social competence (p. 111-124). Maryland, MD: The National Association of School Psychologists.

Eccles, J.S. et Harold, R.D. (1996). Family involvement in children's and adolescents' schooling. In A. Booyh et J.F. Dunn (dir.), Family-school links: How do they affect educational outcomes? (p. 3-34). Mahwah, NJ: Lawrence Erlbaum.

Epstein, J.L. (1995). School/Family/Community Partnerships: Caring for the children we share. Phi Delta Kappan, 76, 701-12.

Fuligni, A.J. (1997). The academic achievement of adolescents from immigrant families: The role of family background, attitudes and behaviour. Child Development, 68(2), 351-363.

Giasson, J. et Saint-Laurent, L. (2004). Le style parental dans les devoirs. Éducation Canada, 45(1), 47-50.

Glasgow Winters, W. (1993). African American mothers and urban schools: The power of participation. New York, NY: Lexington Books.

Henderson, A.T. et Berla, N. (1994). A new generation of evidence: The family is critical to student achievement. Washington, DC: Center for Law and Education.

Henderson, A.T. et Mapp, K.L. (2002). A new wave of evidence: The impact of school, family and community connections on student achievement. Austin, TX: Southwest Educational Development Laboratory.

Holloway, S. (1988). Concepts of ability and effort in Japan and the United States. Review of Educational Research, $58,327-345$.

Hong, S. et Ho, H.-Z. (2005). Direct and indirect longitudinal effects of parental involvement on student achievement: Second-order latent growth modelling across ethnic groups. Journal of Educational Psychology, 97, 32-42.

Humbeeck, B., Lahaye, W., Balsamo, A. et Pourtois, J.-P. (2006). Les relations école-famille: de la confrontation à la coéducation. Revue des sciences de l'éducation, XXXII(3), 649-664.

Institut national d'études démographiques (1970). «Population» et l'enseignement. Paris: Presses universitaires de France.

Johnson, B. et Christensen, L. (2004). Educational research. Quantitative, qualitative, and mixed approaches ( $2^{\circ}$ éd.). Boston, MA: Pearson.

Kelley-Laine, K. (1998). Parents as partners in schooling: The current state of affairs. Childhood Education, 74(6), 342-345.

Lareau, A. (1989). Home Advantage. New York, NY: The Falmer Press.

Legendre, R. (2005). Dictionnaire actuel de l'éducation. Montréal: Guérin.

MacBeth, A. (1989). Involving parents : Effective parent-teacher relations. Heinemann: Oxford.

Martinez, C.R. Jr., DeGarmo, D.S. et Eddy, J. (2004). Promoting academic success among Latino youths. Hispanic Journal of Behavioral Sciences, 26, 128-151.

Montandon, C. (1996). Les relations des parents avec l'école. Lien social et politiques-RIAC, 35, 63-73.

Morrison Gutman, L. et Midgley, C. (2000). The role of protective factors in supporting the academic achievement of poor African American students during the middle school transition. Journal of Youth and Adolescence, 29(2), 223-248.

Okagaki, L. et Frensch, P.A. (1998). Parenting and children's school achievement: A multiethnic perspective. American Educational Research Journal, 35(1), 123-144.

Ong, A.D., Phinney, J.S. et Dennis, J. (2006). Competence under challenge: Exploring the protective influence of parental support and the ethnic identity in Latino college students. Journal of Adolescence, 29, 961-979.

Phinney, J.S., Dennis, J. et Osorio, S. (2006). Reasons to attend college among ethnically diverse college students: Cultural and social class influences. Cultural Diversity and Ethnic Minority Psychology, 12(2), 347-366. 
Pong, S. (1997). Trends in achievement gains: what do we know? Teachers College Record, 99, 23-28.

Potvin, P., Deslandes, R., Beaulieu, P., Marcotte, D., Fortin, L., Royer, É. et Leclerc, D. (1999). Risque d'abandon scolaire, style parental et participation parentale au suivi scolaire. Revue canadienne de l'éducation, 24(4), 441-453.

Saint-Laurent, L., Royer, E., Hébert, M. et Tardif, L. (1994). Enquête sur la collaboration famille-école. Revue canadienne de l'éducation, 19(3), 270-286.

Schneider, M.E. et Ward, D.J. (2003). The role of ethnic identification and perceived social support in Latinos' adjustment to college. Hispanic Journal of Behavioral Sciences, 25, 539-554.

Sheldon, S. et Epstein, J.L. (2005). Involvement counts : Family and community partnerships and mathematics achievement. The Journal of Educational Research, 98(4), 196-206.

Stevenson, H.W., Chen, C. et Uttal, D.H. (1990). Beliefs and achievement: A study of Black, White, and Hispanic children. Child Development, 61, 508-523.

Van der Maren, J.-M. (1996). Méthodes de recherche pour l'éducation (2éd.). Montréal: Les Presses de l'Université de Montréal.

Van Peteghem, O. (2006). Quelles pistes envisager face à la baisse de la participation parentale? Bruxelles: Fédération des associations de parents de l'enseignement officiel.

Van Voorhis, F.L. (2001). Interactive science homeworks : An experiment in home and school connections. NASSP Bulletin, 85, 20-32.

Wigfield, A., Harold, R.D., Freedman-Doan, C., Eccles, J.S., Suk Yoon, K., Arbreton, A. et Blumenfeld, P.C. (1997). Change in children's competence beliefs and subjective task values across the elementary school years. Journal of Educational Psychology, 89, 451-469.

$\mathrm{Xu}, \mathrm{J}$. (2004). Family help and homework management in urban and rural secondary school. Teachers College, 106(9), 1786-1903. 
